\title{
Gesamterneuerungswahlen 2020 der FMH
}

\author{
Jürg Schlup
}

Präsident FMH

\begin{abstract}
Im Rahmen der Ärztekammer vom 7. Mai 2020 finden die Gesamterneuerungswahlen aller Organe der FMH für eine neue Legislatur statt.
\end{abstract}

Gewählt werden:

a) die sieben Mitglieder des Zentralvorstandes der FMH inklusive Präsident und zweier Vizepräsidenten;

b) der Präsident des SIWF;

c) der Präsident und die vier Vizepräsidenten der Standeskommission;

d) die fünf Mitglieder der Geschäftsprüfungskommission;

e) die Mitglieder der Delegiertenversammlung (DV).

Kandidatinnen und Kandidaten für die oben aufgeführten Funktionen a) bis d) haben die Möglichkeit, sich in der Schweizerischen Ärztezeitung in strukturierten Porträts vorzustellen. Bitte beachten Sie, dass die von der Ärztekammer zu wählenden Personen gemäss Art. 22 der Statuten der FMH mit Erreichung ihres 68. Altersjahres aus dem Amt ausscheiden, sofern die Ärztekammer im Einzelfall keine Ausnahme beschliesst.

Ein Anforderungsprofil der Kandidatinnen und Kandidaten für den Zentralvorstand (ZV) sowie das Formular zur Publikation in der SÄZ sind bei den Präsidentinnen und Präsidenten der in der Ärztekammer vertretenen Organisationen oder bei deren Sekretären und Sekretariaten erhältlich. Neben den statutarischen Voraussetzungen (Facharzt-Titel und FMH-Mitgliedschaft) sind unter anderem Voraussetzungen wie politische und standespolitische Erfahrung, Führungskompetenz und Sprachkompetenzen gefordert.

Die Angaben der Kandidatinnen und Kandidaten benötigen wir bis spätestens am 31. Januar 2020 per E-Mail an wahlen2020[at]fmh.ch. Später eintreffende Angaben können nicht mehr in der Ärztezeitung publiziert werden. Ihre Fragen zu den Gesamterneuerungswahlen nehmen wir gerne per E-Mail an wahlen2020[at]fmh.ch entgegen. 